Louisiana State University

LSU Digital Commons

$1-7-2009$

\title{
Monte Carlo fast dose calculator for proton radiotherapy: Application to a voxelized geometry representing a patient with prostate cancer
}

\author{
Pablo Yepes \\ Rice University \\ Sharmalee Randeniya \\ Rice University \\ Phillip J. Taddei \\ University of Texas MD Anderson Cancer Center \\ Wayne D. Newhauser \\ University of Texas MD Anderson Cancer Center
}

Follow this and additional works at: https://digitalcommons.Isu.edu/physics_astronomy_pubs

\section{Recommended Citation}

Yepes, P., Randeniya, S., Taddei, P., \& Newhauser, W. (2009). Monte Carlo fast dose calculator for proton radiotherapy: Application to a voxelized geometry representing a patient with prostate cancer. Physics in Medicine and Biology, 54 (1) https://doi.org/10.1088/0031-9155/54/1/N03 


\title{
Monte Carlo fast dose calculator for proton radiotherapy: application to a voxelized geometry representing a patient with prostate cancer
}

\author{
Pablo Yepes ${ }^{1,3}$, Sharmalee Randeniya ${ }^{1}$, Phillip J Taddei ${ }^{2}$, and Wayne D Newhauser ${ }^{2}$ \\ Pablo Yepes: yepes@rice.edu \\ ${ }^{1}$ Department of Physics and Astronomy, MS 315, Rice University, 6100 Main Street, Houston, TX \\ 77005, USA \\ 2Department of Radiation Physics, Unit 94, The University of Texas M. D. Anderson Cancer \\ Center, 1515 Holcombe Blvd, Houston, TX 77030, USA
}

\section{Abstract}

The Monte Carlo method is used to provide accurate dose estimates in proton radiation therapy research. While it is more accurate than commonly used analytical dose calculations, it is computationally intense. The aim of this work was to characterize for a clinical setup the fast dose calculator (FDC), a Monte Carlo track-repeating algorithm based on GEANT4. FDC was developed to increase computation speed without diminishing dosimetric accuracy. The algorithm used a database of proton trajectories in water to calculate the dose of protons in heterogeneous media. The extrapolation from water to 41 materials was achieved by scaling the proton range and the scattering angles. The scaling parameters were obtained by comparing GEANT4 dose distributions with those calculated with FDC for homogeneous phantoms. The FDC algorithm was tested by comparing dose distributions in a voxelized prostate cancer patient as calculated with well-known Monte Carlo codes (GEANT4 and MCNPX). The track-repeating approach reduced the CPU time required for a complete dose calculation in a voxelized patient anatomy by more than two orders of magnitude, while on average reproducing the results from the Monte Carlo predictions within $2 \%$ in terms of dose and within $1 \mathrm{~mm}$ in terms of distance.

\section{Introduction}

The application of proton radiotherapy as a treatment for cancer has increased substantially in the last decade. Proton treatment provides an enhanced possibility of tumor control while minimizing the likelihood of radiation treatment-related side effects. Several pencil beam algorithms have been developed for proton therapy (Petti 1992, Russell et al 1995, 2000, Hong et al 1996, Schaffner et al 1999). While non-stochastic approaches are fast, their accuracy in heterogeneous media may not be sufficient in some anatomic sites. For example, improved dosimetric accuracy relative to current capabilities may be needed for treatment planning for patients with lung cancer, where an accurate knowledge of the dose distribution

(C) 2009 Institute of Physics and Engineering in Medicine Printed in the UK

${ }^{3}$ Author to whom any correspondence should be addressed. 
throughout the whole lung would be helpful in minimizing the incidence of pneumonitis. However, differences between stochastic and analytical methods were found to be less significant for multi-field treatment (Petti 1996).

An advantage of the stochastic approach is that it can be used to predict the amount of stray and leakage radiation that may emanate from a treatment apparatus. These types of radiation contribute to the risk of radiogenic second cancers; they should be considered in treatment planning, particularly for children with good prospects of long-term survival (Taddei et al 2008). In addition to general-purpose Monte Carlo codes for proton simulation, simplified stochastic methods have been developed (Kohno et al 2003, Fippel and Soukup 2004). Li et al (2005) have reported a track-repeating algorithm that preserves the accuracy of the stochastic approach while substantially reducing computation times. The validation of their results was limited to simple phantoms. The question whether a track repeating algorithm is sufficiently accurate for dose distribution calculations in a voxelized anatomy was addressed previously for a proton beam incident on a voxelized geometry of a patient with prostate cancer (Yepes et al 2009). In this work, two different approaches for the algorithm are explored and applied to a more realistic pair of wide proton beams on a voxelized geometry.

\section{Materials and methods}

\subsection{Database and material information}

A database of proton trajectories in water was generated with GEANT4 (Agostinelli et al 2003). The database consisted of trajectories of particles produced by $251 \mathrm{MeV}$ protons impinging on a $660 \times 660 \times 750 \mathrm{~mm}^{3}$ homogeneous water phantom generated with GEANT4 version 4.8.3 using the default settings of the 'hadrontherapy' example. The dimensions of the phantom were chosen to be large enough so that $99.5 \%$ of the initial energy of the proton was deposited within it. GEANT4, like other particle-transport simulation programs, breaks trajectories into discrete steps. Step magnitude is controlled with the range parameter, which was set to $0.02 \mathrm{~mm}$. For each step, the length, angles relative to the previous step, energy loss and energy deposited were stored in the database. The average number of steps stored per proton was 2045, associated with an average of 326 secondary particles, most of them electrons. The size of the database was $27 \mathrm{kB}$ per proton. The generation of a database with 1 million protons on a cluster with $2.8 \mathrm{GHz}$ Intel-Xeon CPUs required 15 min on 100 CPUs.

The path length per deposited energy $(\mathrm{d} x / \mathrm{d} E)$ in each step was also recorded as a function of energy for protons in water. Similar simulations were also run for homogeneous phantoms of 41 biological materials and some relevant metals, including aluminum and titanium. Distributions differential in energy of relative range, or $R(E)=(\mathrm{d} x / \mathrm{d} E)_{\mathrm{M}} /(\mathrm{d} x / \mathrm{d} E)_{\mathrm{H}_{2} \mathrm{O}}$, depending on the particle energy, were generated for each material, $M$.

\subsection{Fast dose calculation algorithm and parameters}

FDC calculated the dose deposited in the various materials based on the database of particle trajectories in water. This was achieved by scaling the path length of each step and the angle between steps, according to the material and its mass density. The scaling was selected by comparing dose distributions in homogeneous phantoms as calculated by GEANT4 and 
FDC. Two approaches were taken to scale the step length. Method I scaled the step length, $l_{\mathrm{M}}$, for material $\mathrm{M}$, taking into account the energy dependence, based on $R(E)$, with the expression: $l^{\mathrm{M}}=\alpha_{\mathrm{I}}^{\mathrm{M}} \times l^{\mathrm{H}_{2} \mathrm{O}} \times R(E)$, where $\alpha_{\mathrm{I}}^{\mathrm{M}}$ is expected to be close to unity. Method II simply scaled $l_{\mathrm{M}}$ by an energy-independent parameter $\alpha_{\mathrm{II}}^{\mathrm{M}}: l^{\mathrm{M}}=\alpha_{\mathrm{II}}^{\mathrm{M}} l^{\mathrm{H}_{2} \mathrm{O}}$. As a first approximation, $\alpha_{\mathrm{II}}^{\mathrm{M}}$ is inversely proportional to the material density, which is the main parameter driving proton range. For both methods the angle between two steps, $\Phi$, was scaled by an energy-independent parameter, $\beta_{\mathrm{M}}$, according to $\phi_{\mathrm{M}}=\beta_{I(\mathrm{II})}^{\mathrm{M}} \phi_{\mathrm{H}_{2} \mathrm{O}}$. Parameters for methods $\mathrm{I}\left(\alpha_{\mathrm{I}}^{\mathrm{M}}, \beta_{\mathrm{I}}^{\mathrm{M}}\right)$ and $\mathrm{II}\left(\alpha_{\mathrm{II}}^{\mathrm{M}}, \beta_{\mathrm{II}}^{\mathrm{M}}\right)$ were optimized independently by minimizing the $\chi^{2}$ for five proton energies: 50, 100, 150, 200 and $250 \mathrm{MeV}$. The following expression was used to calculate the $\chi^{2}$ for each proton energy:

$$
\chi^{2}(\alpha, \beta)=\frac{1}{3} \sum_{j=x, y, z} \frac{1}{N_{j}} \sum_{i=1}^{N_{j}}\left(\frac{D_{i, j, E}^{R}-D_{i, j, E}^{C}(\alpha, \beta)}{0.01\left(D_{j, E}^{R}\right)^{\max }}\right)^{2}
$$

where $j$ runs over one-dimensional projections (histograms) in each dimension, and $i$ over the bins of the projection histograms; $D^{R}$ and $D^{C}$ refer to the reference dose (GEANT4) and calculated dose (FDC), respectively; $\left(D_{j, E}^{R}\right)^{\max }$ is the maximum value of the reference dose (GEANT4) for projection $j$. The formula used is a $\chi^{2}$-type expression with the bin error fixed to $1 \%$ of the maximum value of the GEANT4 distribution for each energy and projection. This choice gives a larger weight, in the minimization process, to bins with larger deposited energy.

\subsection{Gamma function}

To evaluate the dosimetric accuracy of the FDC distributions in a quantitative manner, we compared them to distributions from GEANT4 using the gamma function, $\Gamma$ (Yepes et al 2009). Two distributions were considered to be sufficiently close when at least $99 \%$ of the voxels had values of $\Gamma_{i}$ smaller than unity.

\subsection{Voxelized phantom and treatment technique}

A voxelized phantom based on the computed tomography images of an actual patient who was treated for prostate cancer at M. D. Anderson Cancer Center was used to compare the results of the GEANT4, MCNPX (Pelowitz 2005), and FDC codes in a clinically realistic scenario. The voxel size was $2.5 \times 2.5 \times 2.5 \mathrm{~mm}^{3}$. Proton beam treatments of the prostate typically utilize a parallel-opposed orientation of two lateral fields; therefore, in this study two circular beams were simulated - one from the left side of the patient and one from the right. Each field was $8 \mathrm{~cm}$ in diameter and mono-energetic (200 MeV).

A total of $10^{8}$ protons were generated with MCNPX, while around 5 million protons were used for GEANT4 and FDC. For the MCNPX simulations, the geometry splitting technique was used to reduce variance in absorbed dose. The patient geometry was cropped to 490 , 680 voxels, thus eliminating many of the voxels outside of the beam path. 
GEANT4 and FDC were run on $2.83 \mathrm{GHz}$ Intel Xeon CPUs, while MCNPX was run on 2.6 GHz AMD64 Opteron CPUs. Xeon processors had access to up to $16 \mathrm{~GB}$ of memory, while the AMD64 processors had $2 \mathrm{~GB}$ of memory. Processing times were scaled to the $2.6 \mathrm{GHz}$ machine, assuming code speed scales with clock frequency.

\section{Results and discussion}

Table 1 shows the values of $\alpha, \beta$ and the average $\chi^{2}$ for the 15 ( 5 energies $\times 3$ projections) distributions used in the minimization for each material in methods I and II for various materials. As expected, the parameter $a_{\mathrm{I}}$ was for all cases very close to unity. The last column of the table shows $a_{\text {II }}$ multiplied by the material density, $\rho$, relative to water density, $\rho \mathrm{H}_{2} \mathrm{O}: \alpha_{\mathrm{II}} \times \rho / \rho_{\mathrm{H}_{2} \mathrm{O}}$. Such a quantity should be close to unity, if the step length scales inversely to density compared to water. For soft tissue the product was close to unity. However, for bone (skeleton materials) and metals it deviated from unity up to $40 \%$. This underscores the advantage afforded by FDC of using more than one type of bone or metal. Average $\chi^{2}$ values below unity were obtained for all materials. In general, method I yielded lower values of $\chi^{2}$, as expected, since the energy dependence is taken into account.

The dose deposited by two opposed $8 \mathrm{~cm}$ wide $200 \mathrm{MeV}$ proton beams on the voxelized phantom of a prostate patient was used to test the FDC algorithm in a clinically realistic scenario. Figure 1 shows the dose for one of the beams along the beam axis $(x)$, as calculated with GEANT4, MCNPX and FDC, for two planes along the $y$-axis. The $y$-axis runs from posterior to anterior of the patient, while the $x$-axis runs from left to right. The distributions are in good agreement for both planes, with a small shift near the Bragg peaks, which generates up to $4 \%$ disagreement in that area, which was estimated to correspond to a 0.5 $\mathrm{mm}$ shift. Cross-field profiles for both beams along the $z$-axis, which runs from inferior to superior of the patient, are depicted in figure 2, for two planes along the $x$-axis. In these plots, FDC and GEANT4 agree to within $4 \%$.

The $\Gamma$ function relative to GEANT4 for each voxel for the dose distribution provides a more quantitative comparison among the various distributions. Figure 3 shows the probability that a voxel's $\Gamma$ is above a certain value for the dose delivered by two opposing beams, where only non-air voxels were included. Both FDC methods yielded similar results, with around 1 in 3000 voxels having a $\Gamma$ value above unity. This indicates that there is good agreement between FDC and GEANT4. Such agreement corresponds to a spatial resolution of less than $1 \mathrm{~mm}$ and a dosimetric accuracy of less than $2 \%$. MCNPX had $0.7 \%$ of the voxels with values above unity, slightly worse than those of FDC. This indicates that the disagreement between FDC and the Monte Carlo on which it is based, GEANT4, is smaller than discrepancies between well-tested Monte Carlo codes in the field, like GEANT4 and MCNPX.

The time to process one history was $1.38 \mathrm{~s}, 0.58 \mathrm{~s}$ and $2.8 \mathrm{~ms}$ for GEANT4, MCNPX and FDC, respectively. 


\section{Conclusions}

Our results indicate that an FDC track-repeating algorithm can dramatically accelerate dose calculations for proton radiotherapy compared to Monte Carlo codes run with standard parameters while preserving dosimetric accuracy. The accuracy of the FDC algorithm was validated by comparing the dosimetric results with GEANT4 and MCNPX. Range scaling implemented with two different methods yielded similar results. Scaling parameters were calculated for 41 biological materials, which will allow the application of the method to more complex geometries including metals, which are relevant for patients with implants. The method was applied to a voxelized phantom that was based on CT images of a patient who was treated for prostate cancer. Better agreement between FDC and GEANT4 was observed than that between MCNPX and GEANT4.

Finally, the FDC approach provided a two orders of magnitude reduction in CPU time while preserving dosimetric accuracy. Unlike GEANT4 and MCNPX, the simulation time per incident particle does not increase in FDC with the number of simulated voxels. This FDC track-repeating algorithm may be applied to other Monte Carlo codes, such as MCNPX and FLUKA $^{11}$ (Fasso et al 2005).

\section{Acknowledgments}

This work was supported in part by the Gulf Coast Center for Computational Cancer Research under contract CABC-2006-5-PY, by Northern Illinois University through a subcontract of the Department of Defense contract W81XWH-08-1-0205 and by the Rice Computational Research Cluster funded by the National Science Foundation grant CNS-0421109 and a partnership between Rice University, Advanced Micro Devices, and Cray.

\section{References}

Agostinelli S, et al. GEANT4 - a simulation toolkit. Nucl Instrum Methods A. 2003; 506:250-303.

Fasso A, Ferrari A, Ranft J, Sala PR. FLUKA: a multi-particle transport code CERN-2005-10, INFN/ TC_05/11, SLAC-R-773. 2005

Fippel M, Soukup M. A Monte Carlo dose calculation algorithm for proton therapy. Med Phys. 2004; 31:2263-73. [PubMed: 15377093]

Hong L, Goitein M, Bucciolini M, Comiskey R, Gottschalk B, Rosenthal S, Serago C, Urie M. A pencil beam algorithm for proton dose calculations. Phys Med Biol. 1996; 41:1305-30. [PubMed: 8858722]

Kohno R, Takada Y, Sakae T, Terunuma T, Matsumoto K, Nohtomi A, Matsuda H. Experimental evaluation of validity of simplified Monte Carlo method in proton dose calculations. Phys Med Biol. 2003; 48:1277-88. [PubMed: 12812446]

Li JS, Shahine B, Fourkal E, Ma CM. A particle track-repeating algorithm for proton beam dose calculation. Phys Med Biol. 2005; 50:1001-10. [PubMed: 15798272]

Pelowitz, DB. MCNPXTM User's Manual. Los Alamos, NM: Los Alamos National Laboratory; 2005. Version 2.5.0 Report LA-CP-05-0369

Petti PL. Differential-pencil-beam dose calculations for charged particles. Med Phys. 1992; 19:13749. [PubMed: 1320182]

Petti PL. Evaluation of a pencil-beam dose calculation technique for charged particle radiotherapy. Int J Radiat Oncol Biol Phys. 1996; 35:1049-57. [PubMed: 8751415]

Russell KR, Grusell E, Montelius A. Dose calculations in proton beams: range straggling corrections and energy scaling. Phys Med Biol. 1995; 40:1031-43. [PubMed: 7659728]

Russell KR, et al. Implementation of pencil Kernel and depth penetration algorithms for treatment planning of proton beams. Phys Med Biol. 2000; 45:9-27. [PubMed: 10661580] 
Schaffner B, Pedroni E, Lomax A. Dose calculation models for proton treatment planning using a dynamic beam delivery system: an attempt to include density heterogeneity effects in the analytical dose calculation. Phys Med Biol. 1999; 44:27-41. [PubMed: 10071873]

Taddei PJ, Mirkovic D, Fontenot JD, Giebeler A, Zheng Y, Kornguth D, Mohan R, Newhauser WD. Stray radiation dose and second cancer risk for a pediatric patient receiving craniospinal irradiation with proton beams. Phys Med Biol. 2009; 54 at press.

Yepes P, Randeniya S, Taddei P, Newhauser W. A track repeating algorithm for fast Monte Carlo dose calculations of proton therapy. Nucl Technol. 2009 at press. 


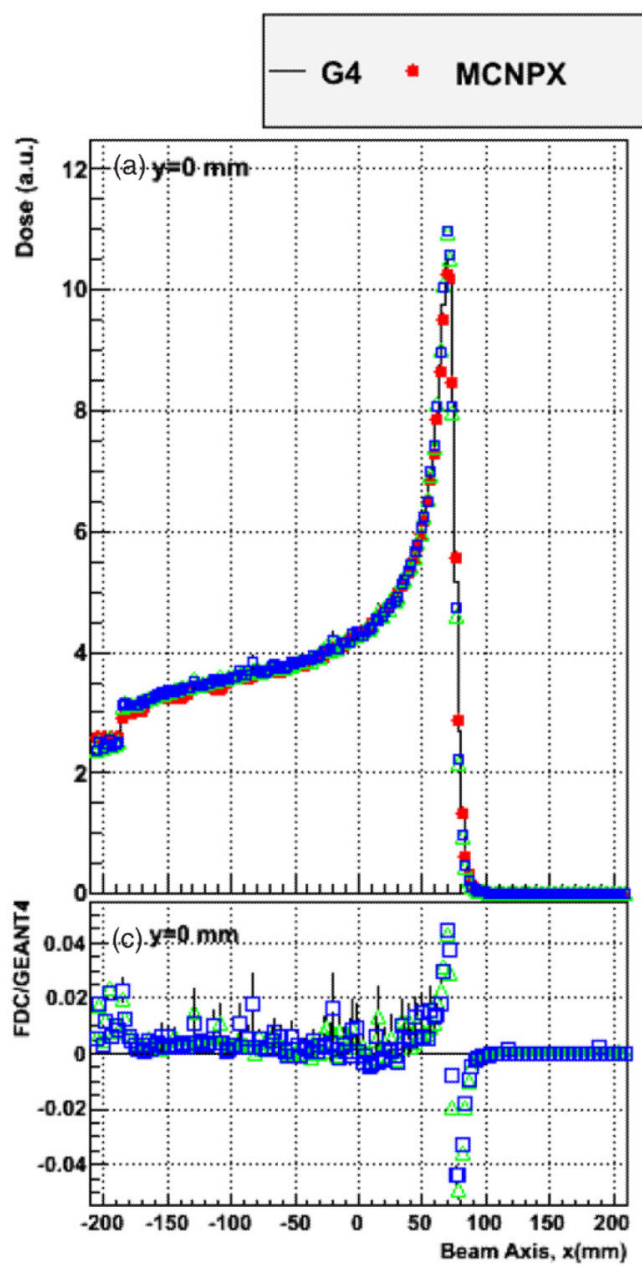

FDC I $\quad$ FDC II

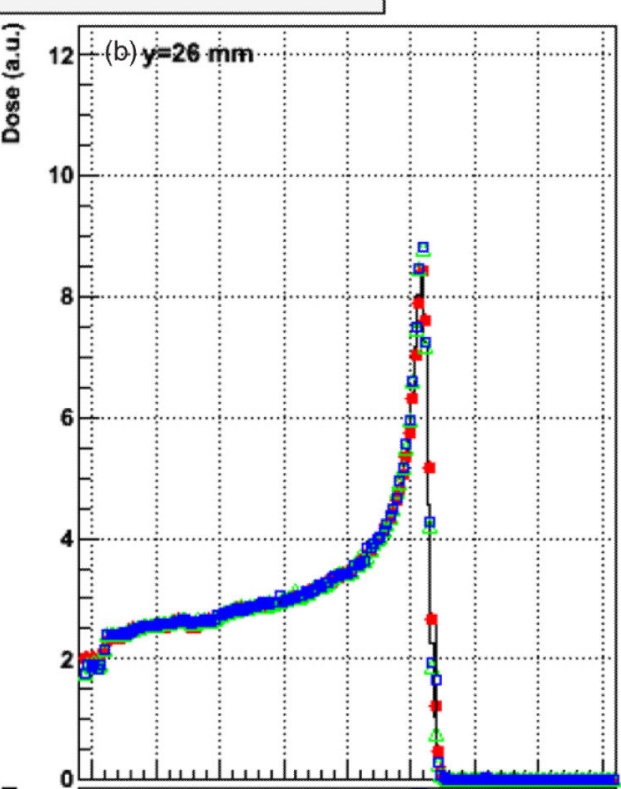

Figure 1.

Dose distributions along the beam axis $(x)$ for one of the $8 \mathrm{~cm}$ diameter circular $200 \mathrm{MeV}$ proton beams for (a) $y=0 \mathrm{~mm}$ and (b) $y=26 \mathrm{~mm}$. The $y$-axis runs from the posterior to anterior of the patient, with its origin at approximately the center of the patient. Distributions were calculated with GEANT4, MCNPX and FDC (methods I and II). The ratios of the FDC to the GEANT4 values are shown in panels (c) and (d) for $y=0 \mathrm{~mm}$ and $y=26 \mathrm{~mm}$, respectively. 


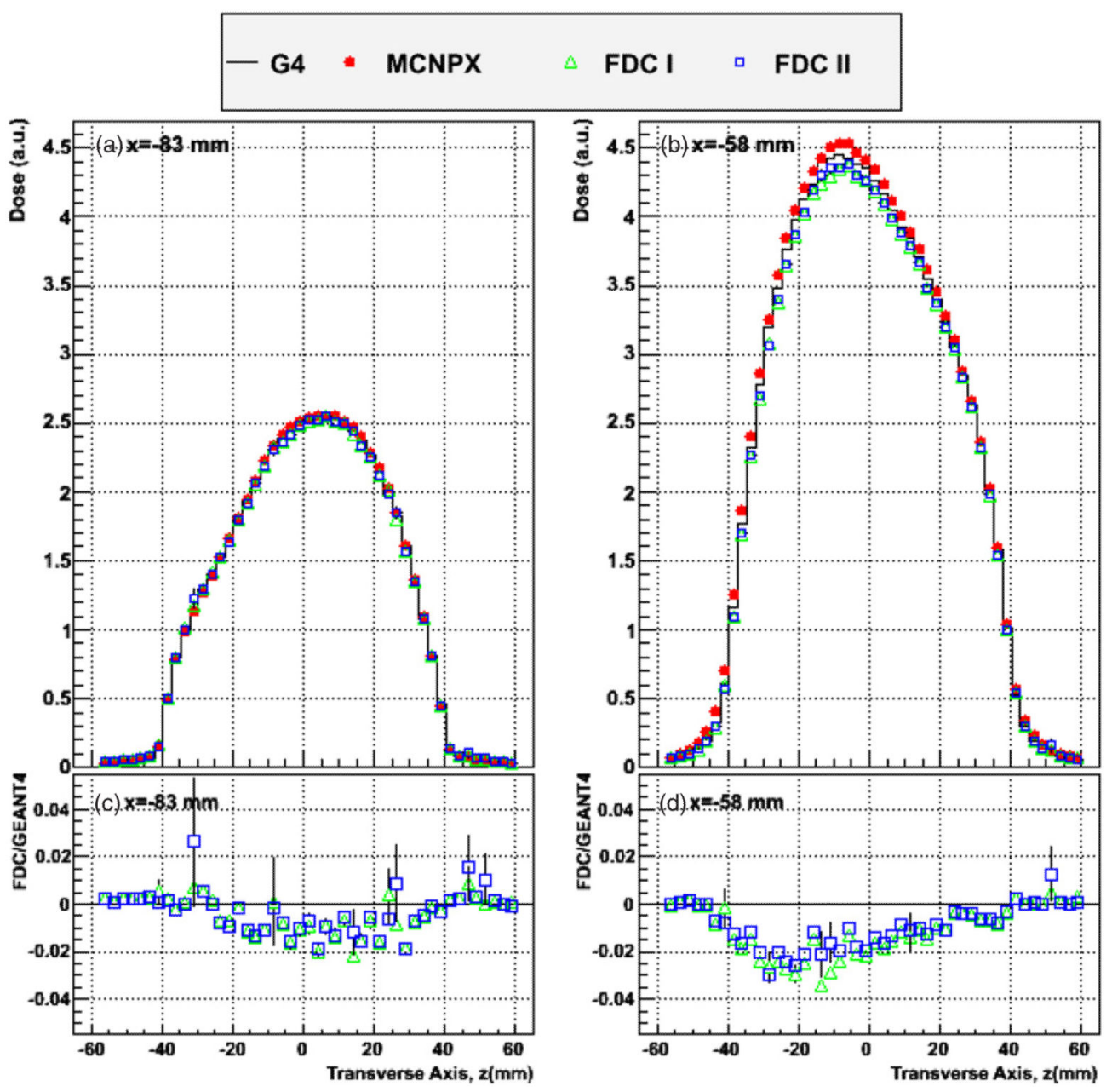

Figure 2.

Dose distributions along the patient vertical axis $(z)$, perpendicular to the beam, for two opposite $8 \mathrm{~cm}$ diameter circular $200 \mathrm{MeV}$ proton beams for (a) $x=-83 \mathrm{~mm}$ and (b) $x=-58$ $\mathrm{mm}$. The $x$-axis corresponds to the beam axis, from the left to the right of the patient, with its origin at approximately the center of the patient. Distributions were calculated with GEANT4, MCNPX and FDC (methods I and II). The ratios of the FDC to the GEANT4 values are shown in panels (c) and (d) for $x=-83 \mathrm{~mm}$ and $x=-58 \mathrm{~mm}$, respectively. 


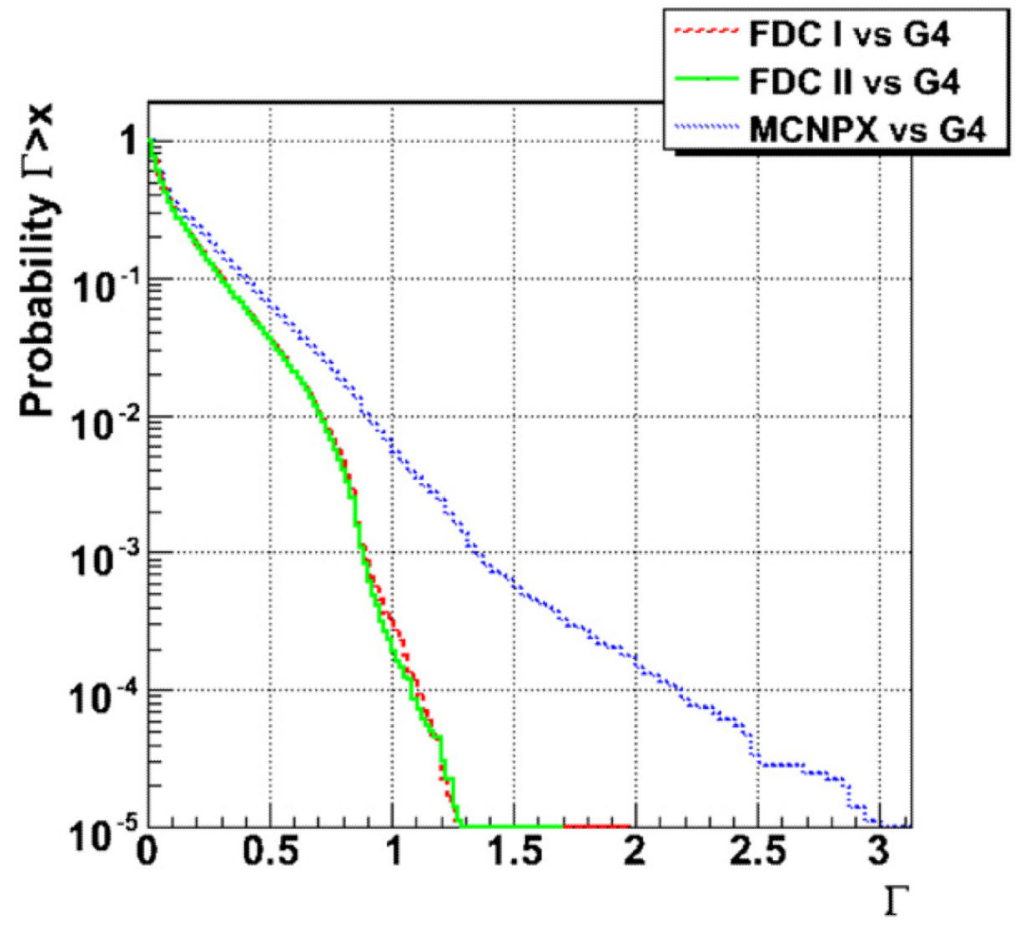

Figure 3.

Voxel probability of having $\Gamma$ above a certain value as a function of $\Gamma$ for FDC (methods I and II) and MCNPX using GEANT4 as reference for the phantom of a patient being treated for prostate cancer with two $8 \mathrm{~cm}$ wide opposing beams of $200 \mathrm{MeV}$ protons. 
$\stackrel{8}{=}$

$\Xi$ 
